\title{
Livestock ownership, animal source foods and child nutritional outcomes in seven rural village clusters in Sub-Saharan Africa
}

Jack B. Hetherington ${ }^{1,2}$, Anke K. Wiethoelter ${ }^{2,3}$, Joel Negin ${ }^{4}$ and Siobhan M. Mor ${ }^{2,3^{*}}$

\begin{abstract}
Background: Sub-Saharan Africa currently has the highest prevalence of malnutrition worldwide. In children under the age of 5 years, malnutrition can have long-term effects on physical and cognitive development, with implications at the national scale. Theoretically, livestock-based interventions are well placed to overcome constraints faced by micronutrient and/or food-based interventions. However, there is limited empirical evidence to support this hypothesis.
\end{abstract}

Methods: This study utilised agriculture, nutrition and anthropometry data from the Millennium Villages Project to investigate relationships between livestock ownership, animal source foods (ASF) consumption and child nutritional outcomes across seven rural village clusters in Sub-Saharan Africa. Village clusters were located in different agro-ecological zones and included: Bonsaaso, Ghana; Mayange, Rwanda; Mwandama, Malawi; Tiby, Mali; Pampaida, Nigeria; Potou, Senegal; and Ruhiira, Uganda. Data from 1624 households (including 1543 children) were included in the analysis.

Results: Overall, the proportion of children with stunting, underweight or wasting across the seven village clusters was 40, 18 and 5\%, respectively. Livestock ownership, ASF consumption and child nutritional outcomes varied between village clusters. Households that owned livestock were generally more likely to consume associated ASF. For example, the proportion of households that consumed milk was higher in households that owned cows compared to those that did not in Pampaida, Mayange and Ruhiira $(P<0.05)$, while poultry meat consumption was generally higher in poultry-keeping households in Mayange and Ruhiira $(P<0.05)$. The relationship between ASF consumption and anthropometric measurements was complex, ranging from positively to negatively associated depending on the food commodity and village cluster. For instance, in Ruhiira, the mean weight-for-age Z score (WAZ) was significantly higher (better) in children from households that consumed eggs in the last 30 days, while in Potou, mean WAZ was significantly lower (worse) in children from households that consumed eggs in the last 30 days $(P<0.05)$.

Conclusions: This study contributes to the growing body of research that investigates the relationships between livestock ownership, ASF consumption and nutritional outcomes in children. Our results reveal complex patterns that vary across agro-ecological zones. More research is needed to assess seasonal variations in these factors, effects of gender roles on intra-household distribution of ASF, as well as effects of zoonotic food-borne diseases on nutritional status of children in these sites.

Keywords: Animal source foods, Child nutrition, Livestock, Sub-Saharan Africa, Millennium Villages Project, One Health

\footnotetext{
*Correspondence: siobhan.mor@sydney.edu.au

${ }^{2}$ Sydney School of Veterinary Science, The University of Sydney,

Camperdown, NSW 2006, Australia

Full list of author information is available at the end of the article
} 


\section{Background}

United Nation's projections indicate that the global population will reach 9.7 billion people by 2050, with a further 1.5 billion people by 2100 [1]. To meet the growing demand for food, it has been estimated that agricultural outputs will need to increase by at least 70\% [2]. Currently, Sub-Saharan Africa has a high prevalence of malnutrition, with approximately half of the world's malnourished human population inhabiting this region [3]. The situation is expected to worsen in the future due to the expected population growth, with countries such as Nigeria and Uganda expected to double their population by 2050 [1].

Malnutrition in children, particularly in children under 5 years of age, can have lasting effects on their physical growth and cognitive development $[4,5]$. Deficiencies in micro- and macro-nutrients [4, 6], recurrent infection [7] and gut dysbiosis [8] can lead to physical manifestations of malnutrition including stunting, underweight or wasting, with extreme cases leading to death. Furthermore, maternal malnutrition has a significant effect on a child's physical and mental development $[6,9]$. The implications of malnutrition also extend beyond the individual, having significant implications for national economies, such as forgone economic productivity caused by partial or total incapacitation from work, including premature death [10].

A range of interventions have been implemented globally to address malnutrition, ranging from micronutrient supplementation programmes (where single micronutrients in the form of injections, formulas or tablets are administered to an individual), to food-based interventions (where nutrient deficiencies are targeted through the provision of different food groups). For example, school-based food programmes have focused on delivery of animal source foods (ASF) including milk and meat to children with some positive gains recorded in terms of improvements to cognitive and physical development [11]. ASF are rich in bioavailable vitamin B12, riboflavin, calcium, zinc, iron and amino acids, and therefore, access to ASF can improve dietary quality [12]. Nevertheless, programmes that rely on government-subsidised micronutrient supplementation or school-based food programmes are unlikely to be sustainable in the long term and may not reach the poorest of households [13].

Evidence is emerging that agricultural interventions can play a role in addressing malnutrition in children. For instance, Palmer et al. [14] demonstrated improved pupillary responsiveness through consumption of provitamin A carotenoid-biofortified maize, while Jones and de Brauw [15] showed promising evidence of orange sweet potato in reducing childhood diarrhoea. While these crop-based interventions have proven to have substantive benefits, to date they have mostly targeted replacement of single micronutrients [16].

Livestock production has the potential to help alleviate food and nutrition insecurity as it not only provides a source of nutrient-dense foods, but also a wide range of saleable commodities and hence a source of financial security and insurance for the rural poor [12, 17, 18]. Benefits from livestock to food and nutrition security arise both directly (by improving household diet through increasing access to ASF) and indirectly (by improving income and ability to purchase more diverse foods) $[18,19]$. With adequate livestock nutrition, renewable ASF, such as eggs and milk, provide an opportunity for a steady supply of essential micro- and macro-nutrients. Indeed, Drewnowski [20] demonstrated that milk and eggs are one of the lowest cost sources of protein amongst plant and animal source foods.

Despite these positive claims, there is limited empirical evidence to support a relationship between livestock production, ASF consumption and improved nutritional outcomes in children. Due to the highly complex nature of food and nutrition insecurity, a One Health approach that specifically aims to bridge the divide between agricultural production and nutrition research is required. This study adapts methods used by Azzarri et al. [21]who performed a detailed analysis of livestock ownership, ASF consumption and child nutritional outcomes in Uganda-to examine these relationships across seven rural village clusters in Sub-Saharan Africa as a basis for informing future nutrition security and livestock-based interventions.

\section{Methods}

This study utilised data from seven rural village clusters participating in the Millennium Villages Project (MVP). The MVP sought 'to demonstrate the feasibility of practical economic transformation in rural tropical Africa through targeted multisectoral investments' [22]. Project sites were chosen to represent the major agro-ecological zones and farming systems in Sub-Saharan Africa [23]. All sites were classified as 'hunger hot spots' where rates of child undernutrition exceeded $20 \%$ at baseline and where the majority of residents were engaged in agriculture [22]. The project included 12 village clusters, across 10 countries in Sub-Saharan Africa, where a cluster included several villages totalling 5000-55,000 people [22]. Sanchez et al. [22] describe village/site selection in more detail.

Data analysed in this study were collected in year 3 of the MVP (2008-2009) and included households from 7 of the 12 village clusters, namely: Bonsaaso, Ghana; Mayange, Rwanda; Mwandama, Malawi; Tiby, Mali; Pampaida, Nigeria; Potou, Senegal; and Ruhiira, Uganda. 
These village clusters were established in 2006 and 2007. By the time of data collection (2008-2009), a few interventions had taken place that likely had some effect on nutritional status. A school meal programme focused on provision of beans, leafy vegetables and soya-enriched maize was implemented in a number of the villages [22]. In addition, provision of fertilizer and improved seed varieties led to increased crop yields in a number of sites [24]. These interventions are unlikely to have had an impact on livestock production and/or ASF consumption. The five remaining village clusters participating in the MVP (Koraro, Ethiopia; Mbola, Tanzania; Dertu, Kenya; Sauri, Kenya; and Ikaram, Nigeria) were not included in this analysis due to differences in questionnaire design and/or comparability of data across countries.

Data used in this study were collected through a series of cross-sectional, face-to-face interviews. The survey modules are described in more detail in the supplementary materials of Sanchez et al. [22] and were largely based on Demographic and Health Surveys (DHS), UNICEF's Multiple Indicator Cluster Surveys (MICS) and the World Bank Living Standards Measurement Study (LSMS) surveys. A household was defined as people who shared the same cooking pot. Questionnaires captured information on household demographics, agriculture and maternal and child health. In addition, questionnaires on food security and food frequency were adapted and piloted at each site and administered to men and women in sampled households (described in more detail in Remans et al. [25]). Finally, anthropometric measurements (height/length, weight and mid-upper arm circumference) were taken on children in participating households.

Food insecurity status was measured as the number of months the respondent/respondents reported as having inadequate food in the past 12 months. We calculated the median (interquartile range, IQR) number of months of food insecurity as well as the proportion of households reporting $\geq 1$ month of food insecurity. Children were classified as being stunted, underweight or wasted on the basis of their height-for-age $Z$ score (HAZ), weightfor-age $Z$ score (WAZ) and weight-for-height $Z$ score (WHZ), respectively, when their score was two standard deviations (SD) below the reference median according to the World Health Organization (WHO) [26]. The mean HAZ/WAZ/WHZ was calculated as well as the proportion of children 2SD below the reference level.

Livestock ownership assessment was based on major food animal species as categorised by the Food and Agriculture Organization of the United Nations (FAO) [23]. Thus, households were classified according to whether they owned one or more of the following: poultry (chicken, ducks and/or other birds), pigs, small ruminants (sheep and/or goats) and cattle (cows, heifers, bulls and/or oxen). Other animals, such as camels, donkeys and horses, were not included as they did not represent a major livestock species across the seven countries.

Food consumption patterns were assessed via a questionnaire, in which an adult member of the household was asked to identify how frequently they consumed specific food items in the last 30 days (none, once per month, 2-3 times per month, once per week, 2-3 times per week, 4-6 times per week, once per day, twice or more per day). For purposes of this analysis, data were re-coded into a binary variable denoting whether or not a particular ASF had been consumed (as main or part of a meal) in the last 30 days. We focused on food commodities produced by the major food animals above, namely: poultry meat, eggs, pork, sheep or goat meat, beef (including veal) and milk and milk products (including those served as a drink or included in sour milk and/or yoghurt). Meat commodities were considered to comprise flesh meat; organ meat (or offal) was not included.

Given diversity of livestock ownership and ASF consumption patterns across the seven sites, we elected to evaluate each site separately. Fisher's Chi-squared (nonparametric) and $t$ tests (parametric) were performed to examine relationships between livestock ownership, ASF consumption and child nutritional status. To adjust for clustering at the household level, mean HAZ/WAZ/ WHZ was calculated for each household. These summary measures were then used in subsequent analyses comparing mean $Z$ scores across households according to the livestock ownership status and ASF consumption patterns. Assessment of ASF consumption patterns in relation to livestock ownership was limited to foods derived from each livestock group (e.g. cattle ownership and beef consumption). Statistical analysis was performed in $\mathrm{R}$ version 3.2.4 (The R Foundation) and SPSS version 22 (IBM, Chicago, IL).

\section{Results}

\section{Household demographics and food security status}

Household demographics are shown in Table 1. A total of 1624 households were included in the analysis. Across the seven countries, $18 \%$ of households had female heads, ranging from less than 1\% (Tiby) to one-third (Mwandama). Approximately $69 \%$ of the respondents did not have any level of formal education. The median household size was 6 people (range 1-60). Of the households surveyed, $47 \%$ reported that they had experienced at least 1 month of food insecurity during the last 12 months. Anthropometric measurements were available for 1543 children belonging to 967 households. The median number of children per household was 1 (range 1-7). The 
Table 1 Household demographic characteristics and food security status by village cluster

\begin{tabular}{|c|c|c|c|c|c|c|c|}
\hline \multirow[t]{2}{*}{ Characteristic } & \multicolumn{7}{|c|}{ Millennium Villages Cluster } \\
\hline & $\begin{array}{l}\text { Bonsaaso, } \\
\text { Ghana }\end{array}$ & $\begin{array}{l}\text { Pampaida, } \\
\text { Nigeria }\end{array}$ & $\begin{array}{l}\text { Potou, } \\
\text { Senegal }\end{array}$ & Tiby, Mali & $\begin{array}{l}\text { Mayange, } \\
\text { Rwanda }\end{array}$ & $\begin{array}{l}\text { Mwandama, } \\
\text { Malawi }\end{array}$ & Ruhiira, Uganda \\
\hline No. of households & 169 & 267 & 274 & 213 & 234 & 245 & 222 \\
\hline No. of children & 145 & 325 & 293 & 210 & 142 & 174 & 254 \\
\hline \multicolumn{8}{|c|}{ Household characteristics } \\
\hline $\begin{array}{c}\text { Household size, } \\
\text { median (IQR) }\end{array}$ & $6(3)$ & $7(4)$ & $9(7)$ & $13(12)$ & $5(2)$ & $5(3)$ & $5(3)$ \\
\hline $\begin{array}{l}\text { Female head of } \\
\text { household, } \\
\text { no. (\%) }\end{array}$ & $40(24)$ & $2(1)$ & $35(13)$ & $1(0)$ & $58(25)$ & $84(34)$ & $65(29)$ \\
\hline \multicolumn{8}{|c|}{ Household head education level, no. (\%) } \\
\hline $\begin{array}{l}\text { No formal } \\
\text { education }\end{array}$ & $73(43)$ & $212(79)$ & $270(99)$ & $210(99)$ & $148(63)$ & $151(62)$ & $163(73)$ \\
\hline Primary school & $31(18)$ & $45(17)$ & $3(1)$ & $1(0)$ & $63(27)$ & $45(18)$ & $41(18)$ \\
\hline $\begin{array}{l}\text { Lower second- } \\
\text { ary school }\end{array}$ & $58(34)$ & $7(3)$ & 0 & $1(0)$ & $8(3)$ & $40(16)$ & $7(3)$ \\
\hline $\begin{array}{l}\text { Upper second- } \\
\text { ary school or } \\
\text { higher }\end{array}$ & $7(4)$ & $3(1)$ & $1(0)$ & $1(0)$ & $15(6)$ & $9(4)$ & $11(5)$ \\
\hline \multicolumn{8}{|l|}{ Food insecurity } \\
\hline $\begin{array}{l}\geq 1 \text { month of } \\
\text { food insecu- } \\
\text { rity, no. (\%) }\end{array}$ & $115(68)$ & $157(59)$ & $140(51)$ & $69(32)$ & $77(33)$ & $102(42)$ & $109(49)$ \\
\hline $\begin{array}{l}\text { No. months of } \\
\text { food insecu- } \\
\text { rity, median } \\
\text { (range) }\end{array}$ & $2(0-12)$ & $1(0-6)$ & $1(0-8)$ & $0(0-6)$ & $0(0-12)$ & $0(0-7)$ & $0(0-12)$ \\
\hline \multicolumn{8}{|c|}{ Child anthropometry } \\
\hline \multicolumn{8}{|c|}{ Mean Z score (SD) } \\
\hline Height-for-age & $-1.40(1.6)$ & $-1.66(2.12)$ & $-1.02(1.83)$ & $-1.49(1.81)$ & $-1.25(1.58)$ & $-1.70(1.47)$ & $-1.81(1.68)$ \\
\hline Weight-for-age & $-1.13(1.24)$ & $-1.01(1.81)$ & $-0.78(1.30)$ & $-1.08(1.32)$ & $-0.73(1.48)$ & $-0.76(1.14)$ & $-0.80(1.26)$ \\
\hline $\begin{array}{l}\text { Weight-for- } \\
\text { height }\end{array}$ & $-0.28(1.10)$ & $-0.24(1.27)$ & $-0.40(1.45)$ & $-0.26(1.25)$ & $-0.09(1.21)$ & $0.34(1.08)$ & $0.42(1.01)$ \\
\hline \multicolumn{8}{|c|}{ Malnourished children, no. (\%) } \\
\hline Stunted & $54(38)$ & $129(47)$ & $75(29)$ & $80(40)$ & $45(32)$ & $72(42)$ & $124(50)$ \\
\hline Underweight & $27(19)$ & $56(26)$ & $46(17)$ & $51(25)$ & $22(15)$ & $17(10)$ & $36(14)$ \\
\hline Wasted & $3(2)$ & $17(8)$ & $33(11)$ & $11(5)$ & $6(4)$ & $2(1)$ & $1(0)$ \\
\hline
\end{tabular}

No. number, $I Q R$ interquartile range, $S D$ standard deviation

proportion of children with stunting, underweight or wasting across the seven village clusters was 40,18 and $5 \%$, respectively.

\section{Livestock ownership patterns}

Livestock ownership patterns were highly variable between village clusters (Table 2). Overall, 77\% (range 44-97\%) of households owned one or more livestock group. The most commonly owned groups in descending order were poultry ( $57 \%$ of households; range $40-81 \%)$, small ruminants (55\%; range $14-85 \%)$, cattle (30\%; range $0-91 \%)$ and pigs (5\%; range $0-21 \%)$. In general, households in this study were smallholders. More than half of households in Bonsaaso and Mwandama owned a single livestock group only (poultry; data not shown). In other sites, mixed livestock farming was practised by a majority of households.

\section{Consumption of animal source foods}

Animal source foods consumption patterns varied considerably across village clusters (Table 3). Overall, $61 \%$ (range 31-84\%) of households reportedly consumed sheep or goat meat as either part of a meal or as the main meal in the past 30 days, 59\% (range 19-86\%) consumed beef, 59\% (range 27-99\%) consumed milk/milk products, $58 \%$ (range $24-77 \%$ ) consumed poultry meat, 57\% (range 
Table 2 Livestock ownership patterns by village cluster

\begin{tabular}{|c|c|c|c|c|c|c|c|c|}
\hline \multirow[t]{2}{*}{ Livestock group } & \multirow[t]{2}{*}{ Unit } & \multicolumn{7}{|c|}{ Millennium Villages Cluster } \\
\hline & & $\begin{array}{l}\text { Bonsaaso, } \\
\text { Ghana }\end{array}$ & $\begin{array}{l}\text { Pampaida, } \\
\text { Nigeria }\end{array}$ & $\begin{array}{l}\text { Potou, } \\
\text { Senegal }\end{array}$ & Tiby, Mali & $\begin{array}{l}\text { Mayange, } \\
\text { Rwanda }\end{array}$ & $\begin{array}{l}\text { Mwandama, } \\
\text { Malawi }\end{array}$ & $\begin{array}{l}\text { Ruhiira, } \\
\text { Uganda }\end{array}$ \\
\hline \multirow[t]{2}{*}{ Cattle } & No. (\%) & 0 & $142(53)$ & $52(19)$ & $193(91)$ & $63(27)$ & 0 & $40(18)$ \\
\hline & Median (range) & NA & $2(1-60)$ & $4(1-105)$ & $4(1-54)$ & $2(1-6)$ & NA & $3(1-15)$ \\
\hline \multirow[t]{2}{*}{ Small ruminants } & No. $(\%)$ & $24(14)$ & $162(61)$ & $232(85)$ & $171(80)$ & $92(39)$ & $58(24)$ & $147(66)$ \\
\hline & Median (range) & $5.5(1-12)$ & $5(1-160)$ & $8(1-160)$ & $5(1-45)$ & $2.5(1-12)$ & $3(1-10)$ & $4(1-23)$ \\
\hline \multirow[t]{2}{*}{ Poultry } & No. (\%) & $68(40)$ & $155(58)$ & $164(60)$ & $173(81)$ & $61(26)$ & $175(71)$ & $133(60)$ \\
\hline & Median (range) & $10(1-50)$ & $9(1-200)$ & $6(1-50)$ & $9(1-340)$ & $4(1-30)$ & $6(1-53)$ & $4(1-19)$ \\
\hline \multirow[t]{2}{*}{ Pigs } & No. $(\%)$ & 0 & 0 & 0 & 0 & $6(3)$ & $27(11)$ & $46(21)$ \\
\hline & Median (range) & NA & NA & NA & NA & $1(1-3)$ & $2(1-7)$ & $1(1-9)$ \\
\hline Any livestock ${ }^{\mathrm{a}}$ & No. (\%) & $74(44)$ & $215(81)$ & $244(89)$ & $206(97)$ & $132(56)$ & $190(78)$ & $187(84)$ \\
\hline
\end{tabular}

Values depict the number (\%) of households that own each livestock group and median (range) herd/flock size in those households that own each livestock group NA not applicable (livestock not owned)

a Any livestock includes poultry, pigs, small ruminants and/or cattle

Table 3 Animal source food consumption patterns, by village cluster

\begin{tabular}{|c|c|c|c|c|c|c|c|}
\hline \multirow[t]{2}{*}{ Animal source food } & \multicolumn{7}{|c|}{ Millennium Villages Cluster } \\
\hline & $\begin{array}{l}\text { Bonsaaso, } \\
\text { Ghana }\end{array}$ & $\begin{array}{l}\text { Pampaida, } \\
\text { Nigeria }\end{array}$ & $\begin{array}{l}\text { Potou, } \\
\text { Senegal }\end{array}$ & Tiby, Mali & $\begin{array}{l}\text { Mayange, } \\
\text { Rwanda }\end{array}$ & $\begin{array}{l}\text { Mwandama, } \\
\text { Malawi }\end{array}$ & Ruhiira, Uganda \\
\hline Beef & $56(33)$ & $230(86)$ & $203(74)$ & $144(68)$ & 135 (58) & 46 (19) & 152 (69) \\
\hline Milk/milk products & $56(33)$ & $179(67)$ & $270(99)$ & $193(91)$ & $115(49)$ & $66(27)$ & $85(38)$ \\
\hline Sheep/goat meat & $52(31)$ & $196(73)$ & $168(61)$ & $178(84)$ & $77(33)$ & $189(77)$ & $131(59)$ \\
\hline Poultry meat & $95(56)$ & $204(76)$ & $190(69)$ & $128(60)$ & $57(24)$ & $188(77)$ & $77(35)$ \\
\hline Eggs & $146(86)$ & $195(73)$ & $231(84)$ & $24(11)$ & $55(24)$ & $205(84)$ & $71(32)$ \\
\hline Pork & $26(15)$ & NA & NA & NA & $21(9)$ & $116(47)$ & $78(35)$ \\
\hline
\end{tabular}

Values depict the number (\%) of households consuming each animal source food (ASF) in the last 30 days

NA not applicable (ASF not included in questionnaire)

$11-86 \%$ ) consumed eggs, and $28 \%$ (range $0-47 \%$ ) consumed pork.

\section{Consumption of animal source foods by livestock ownership status}

Relationships between ASF consumption and livestock ownership status are shown in Table 4. In general, the proportion of households that consumed a particular ASF in the last 30 days tended to be higher in households that owned the livestock group from which the food was sourced compared to households that did not own that livestock group. This was particularly true in Mayange and Ruhiira, where significant positive associations $(P$ value $<0.05)$ existed between ASF consumption and livestock ownership across several commodity-livestock pairings. Of all ASF commodities, the relationship between cattle ownership and dairy consumption was the most consistent across village clusters, with a greater proportion of households that owned cattle reporting that they had consumed milk/milk products in the last 30 days compared to households that did not own cattle. This association was statistically significant in Mayange, Pampaida and Ruhiira $(P$ value $<0.05)$. Similarly, compared to households that did not own poultry, a greater proportion of poultry-keeping households reported eating poultry meat in the last 30 days in four villages clusters, a finding that was statistically significant for Mayange $(P$ value $<0.05)$ and Ruhiira $(P$ value $<0.001)$. Although egg consumption was generally higher in such households, poultry ownership was only significantly associated with higher rates of egg consumption in Mwandama ( $P$ value $<0.05)$.

\section{Livestock ownership and child nutritional status}

The results of the univariate analysis of anthropometric measurements and livestock ownership across the 7 
Table 4 Animal source food consumption, by livestock ownership status and village cluster

\begin{tabular}{|c|c|c|c|c|c|c|c|c|c|c|c|c|c|c|c|}
\hline \multirow[t]{4}{*}{ Animal source food (ASF) } & \multirow[t]{4}{*}{ Livestock group } & \multicolumn{14}{|c|}{ Livestock ownership status (Yes/No) } \\
\hline & & \multicolumn{14}{|c|}{ Millennium Villages Cluster } \\
\hline & & \multicolumn{2}{|c|}{$\begin{array}{l}\text { Bonsaaso, } \\
\text { Ghana }\end{array}$} & \multicolumn{2}{|c|}{$\begin{array}{l}\text { Pampaida, } \\
\text { Nigeria }\end{array}$} & \multicolumn{2}{|c|}{$\begin{array}{l}\text { Potou, } \\
\text { Senegal }\end{array}$} & \multicolumn{2}{|c|}{ Tiby, Mali } & \multicolumn{2}{|c|}{$\begin{array}{l}\text { Mayange, } \\
\text { Rwanda }\end{array}$} & \multicolumn{2}{|c|}{$\begin{array}{l}\text { Mwan- } \\
\text { dama, } \\
\text { Malawi }\end{array}$} & \multicolumn{2}{|c|}{$\begin{array}{l}\text { Ruhiira, } \\
\text { Uganda }\end{array}$} \\
\hline & & Yes & No & Yes & No & Yes & No & Yes & No & Yes & No & Yes & No & Yes & No \\
\hline Beef & Cattle & NA & 33 & 86 & 86 & 75 & 74 & 68 & 60 & 68 & $54^{*}$ & NA & 19 & 68 & 68 \\
\hline Milk/milk products & Cattle & NA & 33 & 73 & $60^{* *}$ & 100 & 98 & 92 & $80^{*}$ & 63 & $44^{* *}$ & NA & 27 & 53 & $35^{* *}$ \\
\hline Sheep/goat meat & Small ruminants & 46 & $28^{*}$ & 71 & 77 & 64 & $48^{*}$ & 82 & 88 & 32 & 34 & 86 & $74^{*}$ & 61 & 56 \\
\hline Poultry meat & Poultry & 56 & 56 & 73 & 81 & 66 & 74 & 61 & 55 & 34 & $21^{* *}$ & 79 & 70 & 45 & $19^{* * *}$ \\
\hline Eggs & Poultry & 87 & 86 & 72 & 75 & 85 & 84 & 13 & 5 & 31 & 20 & 88 & $73^{* *}$ & 36 & 26 \\
\hline Pork & Pigs & NA & 15 & NA & NA & NA & NA & NA & NA & 33 & $8^{*}$ & 44 & 48 & 50 & $31^{* *}$ \\
\hline
\end{tabular}

Values depict the proportion of households that consumed a particular animal source food (ASF) in the last 30 days, stratified by livestock ownership status. Only those ASF that were derived from each livestock group were compared. Proportions that differed significantly (Chi-square test) are indicated with asterisks

$N A$ not applicable (livestock group not owned/ASF not consumed or not included in questionnaire)

* $P<0.1$; ** $P<0.05 ; * * * P<0.001$

village clusters are shown in Fig. 1. Trends varied between villages and across different livestock groups, with few clear patterns. An exception was Pampaida where mean HAZ was significantly higher (better) in children from households that owned cattle or 'any livestock' $(P<0.05)$. In contrast, households that owned 'any livestock' in Mayange had significantly lower (worse) HAZ $(P<0.05)$, suggesting that in this village cluster, owning livestock is a risk factor for child stunting.

\section{Animal source foods consumption and child nutritional status}

The results of the univariate analysis of anthropometric measurements and ASF consumption across the seven village clusters are shown in Fig. 2. Trends again varied between clusters and across different food commodities; however, some consistent patterns emerged for some village clusters. For example, children from households in Mayange and Ruhiira that consumed ASF in the previous

\begin{tabular}{|c|c|c|c|c|c|c|c|c|c|c|c|c|c|c|c|}
\hline \multirow{3}{*}{$\begin{array}{c}Z \\
\text { score }\end{array}$} & \multirow{3}{*}{$\begin{array}{l}\text { Livestock } \\
\text { group }\end{array}$} & \multicolumn{14}{|c|}{ Livestock ownership status (Yes/No) } \\
\hline & & \multicolumn{2}{|c|}{$\begin{array}{c}\text { Bonsaaso, } \\
\text { Ghana }\end{array}$} & \multicolumn{2}{|c|}{$\begin{array}{c}\text { Pampaida, } \\
\text { Nigeria }\end{array}$} & \multicolumn{2}{|c|}{$\begin{array}{l}\text { Potou, } \\
\text { Senegal }\end{array}$} & \multicolumn{2}{|c|}{$\begin{array}{l}\text { Tiby, } \\
\text { Mali }\end{array}$} & \multicolumn{2}{|c|}{$\begin{array}{c}\text { Mayange, } \\
\text { Rwanda }\end{array}$} & \multicolumn{2}{|c|}{$\begin{array}{c}\text { Mwandama, } \\
\text { Malawi }\end{array}$} & \multicolumn{2}{|c|}{$\begin{array}{l}\text { Ruhiira, } \\
\text { Uganda }\end{array}$} \\
\hline & & Yes & No & Yes & No & Yes & No & Yes & No & Yes & No & Yes & No & Yes & No \\
\hline \multirow[t]{5}{*}{ HAZ } & Cattle & NA & -1.46 & -1.43 & $-2.03^{* *}$ & -1.10 & -1.11 & -1.50 & -1.14 & -1.22 & -1.20 & NA & -1.65 & -1.89 & -1.70 \\
\hline & Small ruminants & -1.99 & -1.38 * & -1.53 & -1.94 & -1.11 & -1.10 & -1.43 & -1.67 & -1.42 & -1.02 & -1.40 & -1.72 & -1.73 & -1.77 \\
\hline & Poultry & -1.45 & -1.47 & -1.52 & -1.95 & -1.12 & -1.09 & -1.48 & -1.41 & -1.50 & -1.08 & -1.70 & -1.52 & -1.81 & -1.65 \\
\hline & Pigs & NA & -1.46 & NA & -1.70 & NA & -1.11 & NA & -1.47 & -0.19 & -1.24 & -1.17 & -1.69 & -1.79 & -1.73 \\
\hline & Any livestock ${ }^{1}$ & -1.52 & -1.41 & -1.54 & $-2.40^{* *}$ & -1.12 & -0.96 & -1.47 & -1.51 & -1.46 & $-0.80^{* *}$ & -1.67 & -1.61 & -1.82 & -1.34 \\
\hline \multirow[t]{5}{*}{ WAZ } & Cattle & NA & -1.17 & -1.15 & -1.04 & -0.88 & -0.77 & -1.13 & -0.79 & -0.85 & -0.72 & NA & -0.72 & -0.76 & -0.74 \\
\hline & Small ruminants & -1.29 & -1.15 & -1.17 & -1.00 & -0.78 & -0.90 & -1.10 & -1.09 & -0.93 & -0.60 & -0.58 & -0.75 & -0.70 & -0.82 \\
\hline & Poultry & -1.14 & -1.19 & -1.09 & -1.13 & -0.80 & -0.79 & -1.15 & -0.85 & -0.98 & -0.66 & -0.74 & -0.66 & -0.76 & -0.73 \\
\hline & Pigs & NA & -1.17 & NA & -1.11 & NA & -0.80 & NA & -1.10 & -0.57 & -0.76 & -0.20 & -0.76 & -0.65 & -0.77 \\
\hline & Any livestock ${ }^{1}$ & -1.23 & -1.11 & -1.06 & -1.37 & -0.76 & -1.14 & -1.10 & -1.27 & -0.83 & -0.63 & -0.73 & -0.68 & -0.77 & -0.63 \\
\hline \multirow[t]{5}{*}{$\mathrm{WHZ}$} & Cattle & NA & -0.33 & -0.22 & -0.13 & -0.64 & -0.28 & -0.35 & -0.19 & -0.13 & -0.12 & NA & 0.37 & 0.54 & 0.43 \\
\hline & Small ruminants & -0.81 & -0.26 * & -0.31 & 0.01 & -0.32 & -0.64 & -0.40 & -0.07 & -0.09 & -0.15 & 0.25 & 0.40 & 0.46 & 0.42 \\
\hline & Poultry & -0.19 & -0.44 & -0.19 & -0.16 & -0.43 & -0.24 & -0.36 & -0.22 & -0.33 & -0.04 & 0.38 & 0.34 & 0.51 & 0.37 \\
\hline & Pigs & NA & -0.33 & NA & -0.18 & NA & -0.36 & NA & -0.34 & -0.56 & -0.10 & 0.60 & 0.35 & 0.54 & 0.43 \\
\hline & Any livestock ${ }^{1}$ & -0.31 & -0.36 & -0.17 & -0.22 & -0.32 & -0.80 & -0.34 & -0.64 & -0.12 & -0.12 & 0.36 & 0.40 & 0.47 & 0.33 \\
\hline
\end{tabular}

Fig. 1 Heat map of child anthropometric measurements by livestock ownership status and village. Values represent the mean $Z$ score, adjusted for clustering at the household level. In pairwise comparisons (own livestock/do not own livestock), orange represents lower (worse) $Z$ scores, while blue represents higher (better) $Z$ scores. White indicates no difference between $Z$ scores. ${ }^{*} P<0.1 ;{ }^{* *} P<0.05 ; N A$ not applicable (livestock not owned); ${ }^{1}$ Any livestock includes cattle, small ruminants, poultry and pigs 


\begin{tabular}{|c|c|c|c|c|c|c|c|c|c|c|c|c|c|c|c|}
\hline \multirow{3}{*}{$\begin{array}{c}Z \\
\text { score }\end{array}$} & \multirow{3}{*}{$\begin{array}{l}\text { Animal source } \\
\text { food (ASF) }\end{array}$} & \multicolumn{14}{|c|}{ ASF consumed in the last 30 days (Yes/No) } \\
\hline & & \multicolumn{2}{|c|}{$\begin{array}{c}\text { Bonsaaso, } \\
\text { Ghana }\end{array}$} & \multicolumn{2}{|c|}{$\begin{array}{l}\text { Pampaida, } \\
\text { Nigeria }\end{array}$} & \multicolumn{2}{|c|}{$\begin{array}{l}\text { Potou, } \\
\text { Senegal }\end{array}$} & \multicolumn{2}{|c|}{$\begin{array}{l}\text { Tiby, } \\
\text { Mali }\end{array}$} & \multicolumn{2}{|c|}{$\begin{array}{c}\text { Mayange, } \\
\text { Rwanda }\end{array}$} & \multicolumn{2}{|c|}{$\begin{array}{l}\text { Mwandama, } \\
\text { Malawi }\end{array}$} & \multicolumn{2}{|c|}{$\begin{array}{l}\text { Ruhiira, } \\
\text { Uganda }\end{array}$} \\
\hline & & Yes & No & Yes & No & Yes & No & Yes & No & Yes & No & Yes & No & Yes & No \\
\hline \multirow[t]{6}{*}{ HAZ } & Beef & -1.64 & -1.39 & -1.61 & -2.12 & -1.30 & -0.64 ** & -1.51 & -1.37 & -1.09 & -1.32 & -1.72 & -1.64 & -1.76 & -1.70 \\
\hline & Milk/milk products & -1.54 & -1.43 & -1.57 & -1.94 & -1.13 & 0.19 & -1.57 & -0.58 & -0.92 & $-1.45^{*}$ & -1.83 & -1.57 & -1.53 & -1.91 \\
\hline & Sheep/goat meat & -1.54 & -1.43 & -1.68 & -1.74 & -1.18 & -1.01 & -1.46 & -1.54 & -1.07 & -1.25 & -1.69 & -1.51 & -1.66 & -1.87 \\
\hline & Poultry meat & -1.40 & -1.52 & -1.57 & -2.11 & -1.17 & -0.98 & -1.51 & -1.42 & -1.39 & -1.15 & -1.61 & -1.79 & -1.71 & -1.76 \\
\hline & Eggs & -1.43 & -1.63 & -1.70 & -1.79 & -1.24 & -0.54 ** & -1.37 & -1.48 & -1.33 & -1.17 & -1.62 & -1.87 & -1.53 & -1.85 \\
\hline & Pork & -1.80 & -1.42 & NA & NA & NA & NA & NA & NA & -0.70 & -1.25 & -1.84 & -1.49 & -1.85 & -1.68 \\
\hline \multirow[t]{6}{*}{ WAZ } & Beef & -1.40 & -1.07 & -1.13 & -0.99 & -0.82 & -0.73 & -1.15 & -0.99 & -0.61 & -0.89 & -0.77 & -0.70 & -0.73 & -0.77 \\
\hline & Milk/milk products & -1.14 & -1.18 & -1.09 & -1.13 & -0.80 & -0.45 & -1.19 & -0.30 & -0.57 & -0.91 & -0.73 & -0.71 & -0.60 & -0.85 \\
\hline & Sheep/goat meat & -1.12 & -1.19 & -1.23 & -0.83 & -0.88 & -0.68 & -1.17 & -0.73 & -0.26 & $-0.93^{\star \star}$ & -0.75 & -0.59 & -0.66 & -0.88 \\
\hline & Poultry meat & -1.13 & -1.21 & -1.04 & -1.38 & -0.86 & -0.66 & -1.24 & -0.93 & -0.50 & -0.82 & -0.65 & -0.93 & -0.70 & -0.77 \\
\hline & Eggs & -1.17 & -1.18 & -1.26 & $-0.68 *$ & -0.89 & $-0.38^{* *}$ & -1.21 & -1.08 & -0.57 & -0.79 & -0.70 & -0.84 & -0.49 & $-0.87^{* *}$ \\
\hline & Pork & -1.34 & -1.15 & NA & NA & NA & NA & NA & NA & -0.41 & -0.79 & -0.90 & -0.56 * & -0.73 & -0.75 \\
\hline \multirow[t]{6}{*}{ WHZ } & Beef & -0.61 & $-0.21^{* *}$ & -0.20 & -0.10 & -0.31 & -0.50 & -0.42 & -0.17 & -0.05 & -0.19 & 0.35 & 0.38 & 0.49 & 0.35 \\
\hline & Milk/milk products & -0.29 & -0.35 & -0.20 & -0.14 & -0.36 & -0.45 & -0.38 & 0.01 & -0.20 & -0.05 & 0.39 & 0.36 & 0.50 & 0.40 \\
\hline & Sheep/goat meat & -0.51 & -0.26 & -0.27 & 0.01 & -0.37 & -0.35 & -0.39 & -0.07 & 0.17 & -0.22 & 0.37 & 0.37 & 0.49 & 0.38 \\
\hline & Poultry meat & -0.22 & -0.45 & -0.10 & -0.49 & -0.49 & $-0.10^{*}$ & -0.44 & -0.21 & 0.17 & -0.19 & 0.46 & $0.06^{* *}$ & 0.42 & 0.46 \\
\hline & Eggs & -0.34 & -0.29 & -0.20 & -0.13 & -0.42 & -0.11 & -0.36 & -0.34 & 0.32 & -0.21 * & 0.40 & 0.21 & 0.57 & 0.39 \\
\hline & Pork & -0.48 & -0.31 & NA & NA & NA & NA & NA & NA & 0.04 & -0.14 & 0.22 & 0.51 & 0.49 & 0.42 \\
\hline
\end{tabular}

Fig. 2 Heat map of child anthropometric measurements by consumption of animal source foods (ASF) and village. Values represent the mean $Z$ score, adjusted for clustering at the household level. In pairwise comparisons (ate ASF/did not eat ASF), orange represents lower (worse) $Z$ scores, while blue represents higher (better) $Z$ scores. White indicates no difference between $Z$ scores. ${ }^{*} P<0.1 ;{ }^{*} P<0.05$; NA not applicable (ASF not included in questionnaire)

30 days in general displayed higher (better) anthropometric scores compared to households that did not. In contrast, consumption of ASF tended to be associated with lower (worse) anthropometric scores in children in Potou and Tiby. Mean WAZ was significantly higher (better) amongst children from households that consumed sheep/goat meat or eggs in the last 30 days in Mayange and Ruhiira, respectively $(P<0.05)$. Mean WHZ was also significantly higher amongst children from households in Mwandama that consumed poultry meat in the previous 30 days. In contrast, in Bonsaaso, mean WHZ was significantly lower (worse) amongst children from households that consumed beef in the last 30 days. Mean HAZ was also significantly lower in children from households that consumed beef or eggs in the previous 30 days in Potou.

\section{Discussion}

This study is one of few to provide a comparative, descriptive analysis of the relationships between livestock ownership, ASF consumption and child nutritional outcomes in Sub-Saharan Africa. We found some evidence that owning livestock was associated with increased household consumption of ASF in some study sites; however, owning livestock and/or consuming ASF was not consistently associated with improved child growth in any of the village clusters. Moreover, the results paint a complex picture with considerable variation across sites, highlighting the need to consider local circumstances in any programme promoting livestock development and/ or ASF consumption as a means to alleviate childhood malnutrition.

Farming systems throughout Sub-Saharan Africa are highly variable and dependent on factors such as geography and climate, as well as socio-cultural influences. Across the 15 major farming systems in Sub-Saharan Africa, the contribution of livestock production to rural livelihoods ranges from subsistence or semi-subsistence through to being the primary source of income [23]. Livestock ownership patterns differed considerably across the village clusters, with the proportion of households owning any livestock ranging from less than half in Bonsaaso to nearly all households in Tiby. At least a quarter of households across all seven sites owned poultry, confirming the central role of this livestock species in rural African households [27]. Cattle were important sources of livestock in all but two village clusters: in humid, tropical Bonsaaso, where the presence of tse-tse flies limits the viability of cattle [23], and in Mwandama, where small farm sizes might preclude grazing [28]. Pigs represent an increasingly important sector in Uganda, with $17 \%$ of all households keeping pigs at the recent census [29]. In contrast, the lack of pig production in Nigeria, Mali and Senegal can be explained by the fact that these are predominantly Muslim countries [30]. 
Food consumption patterns were highly variable across the village clusters and likely influenced by many factors beyond whether or not a household owned a particular livestock species (e.g. cultural preferences and different strategies for accessing ASF). A large number of households consumed alternative ASF in addition to those detailed here. For example, 24 and $27 \%$ of households in Pampaida and Potou reported eating camel and tripe in the previous 30 days, respectively. These outcomes were not included in our analysis because they did not represent a significant livestock group or ASF across all sites. Food derived from other animal groups undeniably contributes to total food intake, including fish and fishrelated products [31,32], bush meat [33] and insects [34]. We note that nutrition surveys have tended to focus on skeletal meat and food preferences of western cultures. In the future, efforts to truly understand how ASF contribute to food and nutrition security need to consider the whole animal, in particular offal, and other animal groups such as fish and insects.

Although previous studies have demonstrated a positive association between livestock ownership and ASF consumption [21,35], this is the first study to investigate these relationships across several Sub-Saharan countries. Overall, there were no consistent relationships between livestock ownership and ASF consumption in the preceding 30 days. Our results show that in some village clusters, ASF were significantly more likely to be consumed by households that owned livestock from which the commodity is derived. For instance, we found that cattle, poultry and pig ownership were significantly associated with consumption of milk/milk products, poultry meat and pork, respectively, in Ruhiira, a finding which is consistent with previous studies in Uganda [21]. Similar positive associations were also observed in this study for Mayange, Rwanda. Notably, ASF consumption was amongst the lowest in these village clusters compared to other Millennium Villages. In contrast, poultry, small ruminant and cattle ownership in Tiby were amongst the highest across the 7 village clusters, and the consumption of ASF did not vary significantly between livestock owners and non-owners. This may suggest that there is a threshold effect whereby once a level of livestock ownership is reached in a village, ASF is more readily accessible to all households, including those that do not own a particular livestock species. In other instances, we found consumption of ASF occurred in the absence of a particular livestock species. For example, beef and milk/milk products were consumed with some frequency in Bonsaaso and Mwandama, although no households in these village clusters reported owning cattle. These patterns highlight the complexities of household decision-making regarding food production, trade and consumption [36].
Few studies have investigated the relationship between child nutritional status and livestock ownership and/or ASF consumption in observational studies. Jin and Iannotti [37] found a modest positive association between co-owned/female-owned livestock and WAZ in Kenya, while Mosites et al. [38] found a slight beneficial effect of livestock ownership on stunting prevalence using demographic and health survey data from Ethiopia, Kenya and Uganda. The latter study found no association between ASF consumption (based on 24-h recall) and stunting rates in any country. In this study, we disaggregated ASF into different groups (e.g. poultry meat, pork and eggs), which allowed more detailed analysis of the potential impact of different ASF on child growth. We also captured ASF consumption in the previous 30 days, rather than 24-h recall which does not adequately capture the diversity of a usual diet [39]. Nonetheless, our findings were similar to Mosites et al. [38], in that there was no consistent association between ASF consumption and improved nutritional status in children in any of the village clusters.

There are several limitations of this study. Data analysed in this study are from countries at the very beginning of the nutrition transition [40], with food insecurity and malnutrition still widespread, especially in children [41]. Other research suggests that household diets in Sub-Saharan countries are exposed to large seasonal variety and insecurity around food [42]. It is important to recognise that our study does not account for seasonal variations or extreme weather events (e.g. droughts). Further, it is not representative of national trends as the sample populations were individual villages from the lowest socio-economic bracket and are not representative of rural areas in those countries. Long-term research needs to capture fluctuations in nutritional outcomes, the shifts in livestock ownership and consumption patterns during this transition.

Secondly, interviews were conducted with the household head and we therefore cannot comment on withinhousehold differences in livestock ownership and ASF consumption patterns over the past 30 days. Although 30-day consumption recall has its limitations [43] and is likely to result in bias, we suspect that recall is unlikely to be different between groups (e.g. households that do and do not own livestock), and therefore, any findings would be biased towards the null. Frequency and amount of ASF consumption could plausibly differ, for example, between men, women and children in the household [44, 45]. Given potential intra-household variability of food allocation, particularly between males and females [46], future studies should measure consumption at the individual child level. The importance of women in health and nutritional outcomes of their children is well 
documented [47-49]. For example, Kariuki et al. [48] demonstrated a positive relationship between femaleowned cattle and increased consumption of ASF in Tanzania, Kenya and Mozambique, while Jin and Iannotti [37] found that child HAZ and WAZ scores were higher in households with co-owned/female-owned livestock in Kenya. Future studies are required to investigate how gender roles influence the allocation of food within the household and the impacts on child nutritional outcomes.

Finally, given the high degree of variability across countries and the limited significant findings in the univariate analysis, we elected not to perform further multivariate analysis. Any future study aiming to establish an independent effect of livestock ownership on household food and nutrition security will need to control for factors such as household size, wealth and gender of household head. Additionally, data on breastfeeding and complimentary feeding practices, as well as nutritional status of pregnant or lactating mothers, should be collected and considered in the analysis, given their important influence on child development [50]. Notably, time and labour inputs associated with livestock rearing activities might impact these child feeding practices; more empirical evidence is required to investigate these trade-offs. Access to safe food is also critical for ensuring positive nutritional outcomes in children [6]. Concerns have been raised about possible negative impacts of livestock ownership on child health and development, by increasing infection risks and potentially affecting enteric functions of the gut microbiome [51]. Exposure of infants to livestock faeces has been shown to have a significant impact on bacterial exposure leading to diarrhoea $[52,53]$. There is some evidence that housing of poultry in close proximity to the household can have a negative effect on child growth [54]. Given constraints imposed by the data, we were unable to assess the potential effect of livestock housing, zoonoses and child growth outcomes in the current study.

Food and nutritional security in Sub-Saharan Africa is a complex issue with multiple entry points for creating sustainable improvements. It is plausible that livestock production and ASF can play a role in alleviating hunger, although robust evidence for this impact pathway is still widely lacking. Any intervention will need to be multifaceted, multidisciplinary and tailored to the specific context of the country to ensure a sustained impact is realised. A One Health approach that combines assessment of nutritional outcomes, diet, agricultural and social practices is required. In particular, further detailed research is needed to (1) evaluate the quantity and utilisation of ASF in relation to number of livestock owned by a household; (2) assess the time dimension of food security using longitudinal studies to account for seasonal patterns and changes over time; (3) understand how gender roles influence intra-household variation of food consumption and how livestock ownership may impact this, with emphasis on the most vulnerable (i.e. women and children); and (4) investigate how the potential negative impact of zoonotic/food-borne diseases in households that own livestock or consume ASF can be mitigated to ensure improved nutritional outcomes through livestockbased interventions.

\section{Abbreviations \\ ASF: animal source foods; DHS: Demographic and Health Surveys; FAO: Food and Agriculture Organization of the United Nations; HAZ: height-for-age Z score; IBM: International Business Machines Corporation; IQR: interquartile range; LSMS: Living Standards Measurement Study; MICS: Multiple Indica- tor Cluster Surveys; MVP: Millennium Villages Project; NA: not applicable; SD: standard deviation; SPSS: Statistical Package for the Social Sciences; UN: United Nations; UNICEF: United Nations International Children's Emergency Fund; WAZ: weight-for-age Z score; WHO: World Health Organization; WHZ: weight-for-height $Z$ score.}

\section{Authors' contributions}

JBH performed the analysis, interpreted the data and wrote the manuscript; AKW performed data cleaning, interpreted the data and provided critical evaluation of manuscript drafts; JN conceived of the study idea, facilitated data access and provided critical evaluation of manuscript drafts; and SMM conceived of the study idea, contributed to the analysis, interpreted the data and provided critical evaluation of manuscript drafts. All authors read and approved the final manuscript.

\section{Author details}

${ }^{1}$ Australian Centre for International Agricultural Research, Bruce, ACT 2612, Australia. ${ }^{2}$ Sydney School of Veterinary Science, The University of Sydney, Camperdown, NSW 2006, Australia. ${ }^{3}$ Charles Perkins Centre, The University of Sydney, Camperdown, NSW 2006, Australia. ${ }^{4}$ Sydney School of Public Health, The University of Sydney, Camperdown, NSW 2006, Australia.

\section{Acknowledgements}

The authors would like to thank Roseline Remans, Cheryl Palm and Bocary Kaya from Columbia University for providing the data. Their efforts in the Millennium Villages Project made this study possible. Thanks also to the Australian Centre for International Agricultural Research (ACIAR) for supporting the lead author to undertake this study.

\section{Competing interests}

The authors declare that they have no competing interests.

\section{Availability of data and materials}

Millennium Villages Project data are not publicly available. Access must be negotiated with Columbia University. Data in this study were used under a data-sharing agreement between The University of Sydney and Columbia University. Data that support the findings of this study are available from the authors upon reasonable request and with permission of Columbia University.

\section{Ethics approval and consent to participate}

The study was approved by Institutional Review Boards at Columbia University (AAAA-8202) and in all African partner countries. Community consent was obtained before each survey round in each village cluster. Informed consent was obtained from individuals or parents for all survey procedures.

\section{Funding}

Data used in this study were collected as part of the Millennium Villages Project which was funded by the Lenfest, Blaustein, Sara McCune, Stephen Lewis/ MACAID, and Yara Foundations and the Government of Japan through the United Nations Development Programme's Human Security Trust Fund and 
was headed by Professor Jeffrey Sachs, Earth Institute, Columbia University, USA. The analysis, interpretation of data and writing of the manuscript did not have direct funding.

Received: 2 September 2016 Accepted: 5 December 2016

Published online: 24 January 2017

\section{References}

1. UN. World population prospects revision. New York: United Nations; 2015. p. 2015.

2. FAO. How to feed the world in 2050. Rome: Food and Agriculture Organization of the United Nations; 2009.

3. UNICEF. Levels and trends in child mortality. New York: United Nations International Children's Emergency Fund; 2013.

4. Black MM. Micronutrient deficiencies and cognitive functioning. J Nutr. 2003;133:3927S-31S.

5. Alderman $\mathrm{H}$, Hoddinott J, Kinsey B. Long term consequences of early childhood malnutrition. Oxf Econ Pap. 2006;58:450-74.

6. Müller $\mathrm{O}$, Krawinkel M. Malnutrition and health in developing countries. CMAJ. 2005:173:279-86

7. Schaible UE, Kaufmann SHE. Malnutrition and infection: complex mechanisms and global impacts. PLoS Med. 2007:4:e115.

8. Kane AV, Dinh DM, Ward HD. Childhood malnutrition and the intestinal microbiome malnutrition and the microbiome. Pediatr Res. 2015;77:256-62

9. Osrin D, Vaidya A, Shrestha Y, Baniya RB, Manandhar DS, Adhikari RK, Filteau S, Tomkins A, Anthony MDL. Effects of antenatal multiple micronutrient supplementation on birthweight and gestational duration in Nepal: double-blind, randomised controlled trial. Lancet. 2005:365:955-62.

10. FAO. Incorporating nutrition considerations into development policies and programmes: brief for policy-makers and programme planners in developing countries. Rome: Food and Agriculture Organization of the United Nations; 2004.

11. Whaley SE, Sigman M, Neumann C, Bwibo N, Guthrie D, Weiss RE, Alber S, Murphy SP. The impact of dietary intervention on the cognitive development of Kenyan school children. J Nutr. 2003;133:3965S-71S.

12. Murphy SP, Allen LH. Nutritional importance of animal source foods. J Nutr. 2003;133:3932S-5S.

13. Alders RG, Aongolo A, Bagnol B, de Bruyn J, Kimboka S, Kocj R, Li M, Maulaga W, Mcconchie R, Mor S. Using a One Health approach to promote food and nutrition security in Tanzania and Zambia. Planet@ Risk. 2014;2:187-90.

14. Palmer AC, Healy K, Barffour MA, Siamusantu W, Chileshe J, Schulze KJ, West KP, Labrique AB. Provitamin A carotenoid-biofortified maize consumption increases pupillary responsiveness among Zambian children in a randomized controlled trial. J Nutr. 2016;146:2551-8.

15. Jones KM, de Brauw A. Using agriculture to improve child health: promoting orange sweet potatoes reduces diarrhea. World Dev. 2015;74:15-24.

16. Choi CW. Nutritionally enhanced food crops: progress and perspectives. Int J Mol Sci. 2015;16:3895-914.

17. Moyo S, Swanepoel F. Multifunctionality of livestock in developing communities. In: Swanepoel F, Stroebel A, Moyo S, editors. The role of livestock in developing communities: enhancing multifunctionality. Cape Town: University of the Free State and the Technical Centre for Agricultural and Rural Cooperation; 2010. p. 1-10.

18. Randolph TF, Schelling E, Grace D, Nicholson CF, Leroy JL, Cole DC, Demment MW, Omore A, Zinsstag J, Ruel M. Role of livestock in human nutrition and health for poverty reduction in developing countries. J Anim Sci. 2007:85:2788-800.

19. Smith J, Sones K, Grace D, MacMillan S, Tarawali S, Herrero M. Beyond milk, meat, and eggs: role of livestock in food and nutrition security. Anim Front. 2013;3:6-13.

20. Drewnowski A. The nutrient rich foods index helps to identify healthy, affordable foods. Am J Clin Nutr. 2010;91:1095S-101S.

21. Azzarri C, Zezza A, Haile B, Cross E. Does livestock ownership affect animal source foods consumption and child nutritional status? Evidence from rural Uganda. J Dev Stud. 2015;51:1034-59.

22. Sanchez P, Palm C, Sachs J, Denning G, Flor R, Harawa R, Jama B, Kiflemariam T, Konecky B, Kozar R, Lelerai E, Malik A, Modi V, Mutuo P, Niang
A, Okoth H, Place F, Sachs SE, Said A, Siriri D, Teklehaimanot A, Wang K, Wangila J, Zamba C. The African Millennium Villages. Proc Natl Acad Sci USA. 2007;104:16775-80

23. Dixon JA, Gibbon DP, Gulliver A. Farming systems and poverty: improving farmers'livelihoods in a changing world. Rome: Food and Agriculture Organization of the United Nations; 2001.

24. Denning G, Kabambe P, Sanchez P, Malik A, Flor R, Harawa R, Nkhoma P, Zamba C, Banda C, Magombo C, Keating M, Wangila J, Sachs J. Input subsidies to improve smallholder maize productivity in Malawi: toward an African Green Revolution. PLoS Biol. 2009;7:e1000023.

25. Remans R, Pronyk PM, Fanzo JC, Chen J, Palm CA, Nemser B, Muniz M, Radunsky A, Abay AH, Coulibaly M, Mensah-Homiah J, Wagah M, An X, Mwaura C, Quintana E, Somers MA, Sanchez PA, Sachs SE, McArthur JW, Sachs JD. Multisector intervention to accelerate reductions in child stunting: an observational study from 9 Sub-Saharan African countries. Am J Clin Nutr. 2011;94:1632-42.

26. WHO. Physical status: the use and interpretation of anthropometry. WHO Tech Rep Ser. 1995:854:161-262.

27. de Bruyn J, Wong J, Bagnol B, Pengelly B, Alders R. Family poultry and food and nutrition security. CAB Rev Perspect Agric Vet Sci Nutr Natl Resour. 2015;10:1-9.

28. Goyder H, Mang'anya M. Livestock platform baseline study. Research into Use Malawi Programme-Malawi. 2009.

29. MAAIF. The national livestock census: a summary report of the national livestock census, 2008. Kampala: Ministry of Agriculture, Animal Industry and Fisheries, Uganda and the Uganda Bureau of Statistics; 2009.

30. PRC. The future of the global Muslim population. Washington, DC: Pew Research Centre; 2011.

31. Beveridge M, Thilsted S, Phillips M, Metian M, Troell M, Hall S. Meeting the food and nutrition needs of the poor: the role of fish and the opportunities and challenges emerging from the rise of aquaculture. J Fish Biol. 2013;83:1067-84

32. Verduzco-Gallo I, Ecker O, Pauw K. Changes in food and nutrition security in Malawi. Washington, DC: International Food Policy Research Institute; 2014.

33. Loibooki M, Hofer H, Campbell KL, East ML. Bushmeat hunting by communities adjacent to the Serengeti National Park, Tanzania: the importance of livestock ownership and alternative sources of protein and income. Environ Conserv. 2002;29:391-8.

34. Belluco S, Losasso C, Maggioletti M, Alonzi CC, Paoletti MG, Ricci A. Edible insects in a food safety and nutritional perspective: a critical review. Compr Rev Food Sci Food Saf. 2013;12:296-313.

35. Leroy JL, Frongillo EA. Can interventions to promote animal production ameliorate undernutrition? J Nutr. 2007;137:2311-6.

36. Schaetzel T, Antal M, Guyon A. Household decision-making on homestead food production: perceptions on planting, production, and purchases in Bangladesh. Arlington: United States Agency for International Development; 2014.

37. Jin M, lannotti LL. Livestock production, animal source food intake, and young child growth: the role of gender for ensuring nutrition impacts. Soc Sci Med. 2014;105:16-21.

38. Mosites EM, Rabinowitz PM, Thumbi SM, Montgomery JM, Palmer GH, May S, Rowhani-Rahbar A, Neuhouser ML, Walson JL. The relationship between livestock ownership and child stunting in three countries in Eastern Africa using national survey data. PLoS ONE. 2015;10:e0136686.

39. Barrett-Connor E. Nutrition epidemiology: how do we know what they ate? Am J Clin Nutr. 1991;54:182S-7S.

40. Haggblade S, Duodu KG, Kabasa JD, Minnaar A, Oijo NK, Taylor JR. Emerging early actions to bend the curve in Sub-Saharan Africa's nutrition transition. Food Nutr Bull. 2016:37:219-41.

41. FAO. The state of food insecurity in the World. Rome: Food and Agriculture Organization of the United Nations; 2015.

42. Hirvonen K, Taffesse AS, Worku Hassen I. Seasonality and household diets in Ethiopia. Public Health Nutr. 2016;19:1723-30.

43. Beegle K, De Weerdt J, Friedman J, Gibson J. Methods of household consumption measurement through surveys: experimental results from Tanzania. J Dev Econ. 2012;98:3-18.

44. Bargain O, Donni O, Kwenda P. Intrahousehold distribution and poverty: evidence from Côte d'Ivoire. J Dev Econ. 2014;107:262-76.

45. Guèye EF. The role of family poultry in poverty alleviation, food security and the promotion of gender equality in rural Africa. Outlook Agric. 2000;29:129-36. 
46. Jones AD, Ngure FM, Pelto G, Young SL. What are we assessing when we measure food security? A compendium and review of current metrics. Adv Nutr. 2013;4:481-505.

47. Kassie M, Ndiritu SW, Stage J. What determines gender inequality in household food security in Kenya? Application of exogenous switching treatment regression. World Dev. 2014;56:153-71.

48. Kariuki J, Njuki J, Mburu S, Waithanji E. Women, livestock ownership and food security. Nairobi: International Livestock Research Institute and International Development Research Centre; 2013.

49. Valdivia C. Gender, livestock assets, resource management, and food security: lessons from the SR-CRSP. Agric Hum Values. 2001;18:27-39.

50. Wu G, Bazer FW, Cudd TA, Meininger CJ, Spencer TE. Maternal nutrition and fetal development. J Nutr. 2004;134:2169-72.

51. Mosites E, Mwangi T, Otiang E, Garland-Lewis G, Sammons M, Onyango C, Eng A, Noecker C, Manor O, Hilton S, Call D, Kariuki N, Zambriski J, Wasserheit J, Walson J, Palmer G, Montgomery J, Borenstein E, Omore $R$, Rabinowitz P. Characterising the taxonomic composition of children and livestock gut microbiomes and of environmental samples and the potential role for household-level microbiome sharing in western Kenya. Lancet Glob Health. 2016;4:S20.

52. Ngure FM, Humphrey JH, Mbuya MNN, Majo F, Mutasa K, Govha M, Mazarura E, Chasekwa B, Prendergast AJ, Curtis V, Boor KJ, Stoltzfus RJ. Formative research on hygiene behaviors and geophagy among infants and young children and implications of exposure to fecal bacteria. Am J Trop Med Hyg. 2013;89:709-16.

53. Checkley W, Buckley G, Gilman RH, Assis AM, Guerrant RL, Morris SS, Molbak K, Valentiner-Branth P, Lanata CF, Black RE. Multi-country analysis of the effects of diarrhoea on childhood stunting. Int J Epidemiol. 2008:37:816-30.

54. Headey D, Hirvonen K. Exploring child health risks of poultry keeping in Ethiopia: insights from the 2015 Feed the Future survey. Washington, DC: International Food Policy Research Institute and Ethiopian Development Research Institute; 2015

\section{Submit your next manuscript to BioMed Central and we will help you at every step:}

- We accept pre-submission inquiries

- Our selector tool helps you to find the most relevant journal

- We provide round the clock customer support

- Convenient online submission

- Thorough peer review

- Inclusion in PubMed and all major indexing services

- Maximum visibility for your research

Submit your manuscript at www.biomedcentral com/submit 